\title{
Acromegalia com Níveis Séricos Basais do Hormônio do Crescimento Dentro dos Limites da Normalidade
}

\author{
Mônica P. da Costa \\ Márcia C. da Silva \\ Alice Helena D. Violante \\ Denise C.L. Martins \\ Honomar F. Souza
}

\author{
Hospital Universitário Antônio Pedro \\ - Disciplina de Endocrinologia, \\ Universidade Federal Fluminense \\ (UFF), Niterói, RJ e Hospital \\ Universitário Clementino Fraga Filho \\ - Serviço de Endocrinologia, \\ Universidade Federal do Rio de \\ Janeiro (UFRJ), Rio de Janeiro, RJ.
}

\begin{abstract}
RESUMO
A acromegalia é uma sindrome causada pela hipersecreção do hormônio de crescimento $(\mathrm{GH})$. No entanto, alguns pacientes podem apresentar niveis séricos limítrofes, ou mesmo dentro da normalidade, dificultando o diagnóstico. Relatamos um caso de acromegalia cuja investigação diagnóstica inicial evidenciou níveis séricos basais de $\mathrm{GH}$ dentro dos limites da normalidade e tomografia computadorizada (TC) de sela túrcica normal. A confirmação da doença só foi estabelecida através de testes dinâmicos que avaliam a regulação do $\mathrm{GH}$ (dosagem de $\mathrm{GH}$ durante o teste oral de tolerância à glicose e após TRH) e confirmada pela ressonância nuclear magnética (RNM) de sela túrcica que evidenciou imagem sugestiva de microadenoma. A paciente foi submetida à cirurgia hipofisária pela via de acesso oronaso esfenoidal e a área tumoral encaminhada para estudo imunohistoquímico, corroborando 0 diagnóstico de acromegalia. A avaliação dinâmica no pós operatório evidenciou regulação normal do $\mathrm{GH}$. Discutimos os critérios diagnósticos da acromegalia, com ênfase na importância de se prosseguir com a investigação sempre que houver uma forte suspeita clínica, mesmo que os exames iniciais se apresentem dentro dos limites da normalidade.
\end{abstract} (Arq Bras Endocrinol Metab 2000;44/5: 434-439)

Unitermos: Acromegalia; GH basal normal; Tumor de hipófise; Cirurgia transesfenoidal

\begin{abstract}
Acromegaly is a syndrome caused by growth hormone ( $\mathrm{GH}$ ) hypersecretion. However, some patients can present normal or near normal levels of $\mathrm{GH}$, difficulting diagnosis. We report an acromegalic patient with normal levels of $\mathrm{GH}$ and negative imaging at initial investigation. Acromegaly was confirmed by dynamic tests (glucose tolerance test and $\mathrm{GH}-\mathrm{TRH}$ ), and RMI was suggestive of a microadenoma. The patient was submitted to a transesphenoidal pituitary surgery and imunohistochemistry study, corroborating the diagnosis of acromegaly. Dynamic tests performed after surgery evidenced normal $\mathrm{GH}$ regulation. Considerations on the diagnosis of acromegaly were also done, emphasizing the importance of continuing the investigation whenever clinical suspicion persists, even though the initial tests are within the normal range.
\end{abstract}

(Arq Bras Endocrinol Metab 2000;44/5: 434-439)

Keywords: Acromegaly; Normal basal GH: Hipofise tumor; Transesfenoidal surgery

\section{RELATO DO CASO}

I .F.R., FEMinina, PARDA, 36 anos, Natural da Paraíba, residente no Rio de Janeiro há 10 anos, procurou o Serviço de Endocrinologia do Hospital Universitário Antônio Pedro em julho de 1996 com queixas de aparecimento de pêlos no rosto e nos membros inferiores nos últimos 4 anos,
Recebido em 01/07/99

Revisado em $27 / 04 / 00$

Aceito em 12/05/00 
Tabela 1. Dosagens hormonais basais no pré operatório.

\begin{tabular}{lccccccc}
\hline Hormônio & IGF-BP3 & IGF-1 & GH & LH & FSH & TSH & PRL \\
& $(\mathrm{mg} / \mathrm{l})$ & $(\mathrm{Ul} / \mathrm{ml})$ & $(\mathrm{ng} / \mathrm{ml})$ & $(\mathrm{Ul} / \mathrm{ml})$ & $(\mathrm{Ul} / \mathrm{ml})$ & $(\mu U \mathrm{ml} / \mathrm{ml})$ & $(\mathrm{ng} / \mathrm{ml})$ \\
& 5,36 & 2,52 & 4,4 & 6,10 & 4,30 & 0,67 & 68,69 \\
V.R. & $2,3-5,9$ & $0,5-2,2$ & $0-7$ & $0,4-15,6$ & $1,2-11,7$ & $0,6-6,2$ & $1,3-2,5$ \\
\hline
\end{tabular}

associadas à galactorréia bilateral espontânea um ano após o início do quadro. Referia ciclos menstruais regulares durante este período sem uso de contraceptivos orais e ganho de $15 \mathrm{~kg}$ nos últimos 3 anos. Negava uso de medicações, cefaléia, alteração de campo visual ou estimulação mamária. Ao ser abordada, referiu aumento das extremidades distais, com perda da aliança de casamento e mudança de um número no sapato.

Ao exame físico notava-se embrutecimento do fácies, com aumento do arco zigomático; prognatismo discreto sem separação da arcada dentária; espessamento da pele, mas sem oleosidade excessiva ou acne; hirsutismo moderado em face, nádegas, linha alba e membros inferiores, necessitando depilar 1 a 2 vezes por semana; galactorréia bilateral à expressão. Hidratada, corada, normotensa, com freqüências cardíaca e respiratória normais, sem outra anormalidade ao exame clínico ou visceromegalias (1).

A investigação laboratorial inicial revelou valores normais de glicose, cálcio e fósforo séricos e urinários, clearance de creatinina, ritmo de cortisol e hormônios tireoideanos. Os níveis séricos hormonais basais encontram-se na tabela 1. A ultrassonografia pélvica e a tomografia computadorizada (TC) de sela túrcica foram normais. $\mathrm{O}$ índice calcâneo plantar era de $28 \mathrm{~mm}$.

Em virtude da forte suspeita clínica, a paciente foi internada para realização de testes dinâmicos da regulação do $\mathrm{GH}$, apesar dos exames inicias não evidenciarem hipersecreção deste hormônio. As dosagens de GH durante o TOTG não evidenciaram supressão do mesmo; houve um aumento paradoxal nos niveis de GH após infusão de $500 \mathrm{mcg}$ de TRH; a colheita de $\mathrm{GH}$ durante 24 horas mostrou valores médios entre 5,7 e $12,5 \mathrm{ng} / \mathrm{ml}(\mathrm{VN}=0-7 \mathrm{ng} / \mathrm{ml})$; a avaliação dinâmi-

Tabela 2. Dosagem de GH após $100 \mathrm{~g}$ de dextrosol (TOTG) no pré-operatório.

\begin{tabular}{ccc}
\hline TEMPO & GH $(\mathrm{ng} / \mathrm{ml})$ & Glicose $(\mathrm{mg} / \mathrm{dl})$ \\
\hline Basal & 5,2 & 92 \\
$60^{\circ}$ & 6,0 & 113 \\
$120^{\circ}$ & 4,7 & 97 \\
V.R. & $0-7$ & $70-110$ \\
\hline
\end{tabular}

ca da função hipotálamo-hipofisária (megateste) e o teste de estímulo de GH com L-dopa não evidenciaram alterações significativas.

O ecocardiograma, a campimetria visual, o teste da cortrosina e as provas de função pulmonar foram normais. A ressonância nuclear magnética (RNM) de sela túrcica evidenciou hipófise de volume aumentado, com nódulo hipodenso, não captante, em lobo anterior direito, medindo 0,6 x $0,8 \mathrm{~cm}$, compatível com microadenoma, e haste hipofisária discretamente desviada para a esquerda.

A paciente foi submetida à hipofisectomia por via transoronasoesfenoidal e a análise imunohistoquímica do material revelou adenoma de hipófise pluri-hormonal com imunoexpressão de GH (45\% das células), prolactina (35\%), sub-unidade $\alpha(30 \%)$, FSH (20\%) e ACTH (5\%).

No pós operatório a paciente foi novamente reavaliada. As dosagens de GH basal e com o TOTG não mais apresentavam evidências de hipersecreção do $\mathrm{GH}$. Dois anos após a cirurgia a paciente encontra-se assintomática, com melhora do espessamento da pele e atenuação do embrutecimento do fácies, havendo também uma perda ponderal de $8 \mathrm{~kg}$.

\section{DISCUSSĀO}

A acromegalia é uma sindrome bem definida, causada pela hipersecreção do hormônio do crescimento (GH), com conseqüente elevação dos niveis plasmáticos do fator de crescimento semelhante à insulina (IGF-I) em indivíduos após a puberdade; antes da puberdade ocasiona o gigantismo (2).

Em geral a hipersecreção do $\mathrm{GH}$ é proveniente das células somatotróficas da hipófise anterior, que por

Tabela 3. Dosagem de GH após $500 \mu g$ de TRH no pré-operatório.

\begin{tabular}{ccc}
\hline TEMPO & GH $(\mathrm{ng} / \mathrm{ml})$ & $\mathrm{PRL}(\mathrm{ng} / \mathrm{ml})$ \\
\hline Basal & 4,4 & 54,93 \\
$15^{\prime}$ & 25,2 & 224,44 \\
$30^{\prime}$ & 29,2 & 202,33 \\
$45^{\prime}$ & 18,3 & 135,27 \\
$60^{\prime}$ & 17,0 & 109,68 \\
$90^{\prime}$ & 10,7 & 73,15 \\
$120^{\prime}$ & 7,6 & 59,14 \\
V.R. & $0-7$ & $1,3-25$ \\
\hline
\end{tabular}


Tabela 4. Megateste.

\begin{tabular}{lccccc}
\hline & Basal & $30^{\prime}$ & $60^{\prime}$ & $90^{\prime}$ & $120^{\prime}$ \\
\hline FSH (Ul/ml) & 2,3 & 8,8 & 9,6 & 9,5 & 83,0 \\
LH (Ul/ml) & 1,8 & 44,5 & 29,2 & 23,3 & 19,0 \\
GH (ng/ml) & 5,1 & 32,0 & 20,0 & 17,0 & 8,8 \\
TSH (Ul/ml) & 1,1 & 14,8 & 8,09 & 7,36 & 4,59 \\
PRL (ng/ml) & 52,5 & 133,5 & 75,6 & 68,9 & 56,56 \\
Cortisol (ng/ml) & 107 & 76,3 & 75,0 & 91,0 & 59,0 \\
Glicose (mg/dl) & 96 & 53 & 55 & 81 & 102 \\
\hline
\end{tabular}

VR: FSH: 1,8-1 1,7; LH: 0,4-15,5; GH: 0-7; TSH: 0,6-6,2; PRL: 1,3-25; Cortisol: 5-20; Glicose: 70110.

um defeito intrínseco ocasiona a ativação de um fator de crescimento celular ou a inativação de um fator inibidor de proliferação celular, levando ao aparecimento de um adenoma funcionante autônomo (3). Alguns tumores hipofisários secretores de GH contém mutações somáticas (mutação Gsp) que mimetizam o estímulo do GHRH, mantendo os niveis de AMPc constantemente elevados dentro das células produtoras de GH (3).

$\mathrm{O}$ estudo imunohistoquímico destes tumores revela grânulos secretores de $\mathrm{GH}$ isolados ou em associação com células produtoras de outros hormônios, sendo o mais freqüente destes a prolactina (4).

Devido à evolução insidiosa da doença, o diagnóstico pode ser retardado em 10 a 15 anos, com o aparecimento de queixas inespecíficas e fazendo com que o paciente procure diferentes especialistas até se estabelecer o diagnóstico, quase sempre suspeitado quando as alterações fenotípicas características estão evidentes $(1,4)$. Alguns pacientes jovens, no entanto, podem apresentar um crescimento tumoral rápido, com valores de GH bastante elevados, e, logo no início da doença, apresentarem manifestações neurológicas devido ao efeito massa do tumor causado pela expansão supra selar do mesmo (3).

A incidência anual da acromegalia é de 3 a 4 casos novos por milhão e uma prevalência anual de 50-70 casos por milhão. Está associada a um aumento na mortalidade de cerca de 2 a 3 vezes em relação à população geral, tendo como principais causas de morte: cardiovasculares $(38-62 \%)$, respiratórias $(0$ $25 \%$ ) e neoplásicas (9-25\%) (3-5). Acomete mais o sexo feminino, entre a $3^{\text {a }}$ e $4^{\text {a }}$ décadas de vida (4).

Tabela 5. Dosagens hormonais basais no pós operatório.

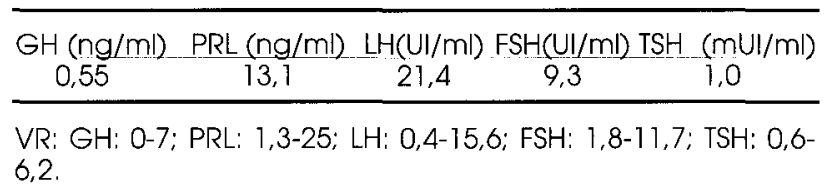

Semelhante ao ritmo fisiológico, a secreção do $\mathrm{GH}$ pelos adenomas ocorre de maneira pulsátil, porém a sua freqüência e amplitude encontram-se aumentadas. No entanto, alguns pacientes podem apresentar valores laboratoriais limítrofes ou mesmo dentro da normalidade, o que dificulta ainda mais o diagnóstico $(6,7)$. Tais casos são relativamente raros, representando cerca de $3,7 \%$ dos casos na maioria dos estudos $(7,8)$. Assim sendo, uma única dosagem do GH basal não constitui um marcador confiável para o diagnóstico ou exclusão da acromegalia; testes dinâmicos que avaliam a regulação do GH devem sempre ser realizados para confirmação diagnóstica (3,9-11).

A paciente acromegálica, neste caso, apresentava níveis séricos basais de $\mathrm{GH}$ menor do que $5 \mathrm{ng} / \mathrm{ml}$, o que é considerado dentro da faixa da normalidade (6) e tomografia computadorizada de sela túrcica normal. Em muitos centros especializados a investigação terminaria neste estágio. Entretanto, existe um número crescente de estudos indicando que a acromegalia ativa pode estar associada a níveis séricos de GH aparentemente baixos, e tais casos devem ser distinguidos através de testes dinâmicos de regulação do $\mathrm{GH}(7,8)$. O diagnóstico da paciente em questão só foi estabelecido através da realização dos testes dinâmicos que evidenciaram hipersecreção de GH e confirmado pela ressonância nuclear magnética (RNM) de sela túrcica, que evidenciou imagem compatível com microadenoma hipofisário. A RNM parece constituir o exame de imagem de escolha nestes pacientes. Raramente uma massa hipofisária não é detectada por este método e, nestes casos, deve-se considerar a presença de um tumor ectópico secretante de GH ou de GHRH (3).

Tabela 6. Dosagem de GH após $100 \mathrm{~g}$ de dextrosol (TOTG) no pós operatório.

\begin{tabular}{lcc}
\hline TEMPO & $\mathrm{GH}(\mathrm{ng} / \mathrm{ml})$ & Glicose $(\mathrm{mg} / \mathrm{dl})$ \\
Basal & 1,1 & 96 \\
$60^{\prime}$ & 1,8 & 119 \\
$120^{\prime}$ & 1,4 & 98 \\
\hline
\end{tabular}

VR: GH:0-7; Glicose: 70-110. 
Dentre os testes dinâmicos utilizados, a dosagem do GH após administração via oral de $100 \mathrm{~g}$ de dextrosol, parece ser o gold standard. Em todos os indivíduos normais ocorre supressão dos níveis de GH para valores abaixo de $2 \mathrm{ng} / \mathrm{ml}$ (7). Esse fenômeno não acontece nos pacientes com acromegalia em atividade; ao contrário, pode haver um aumento paradoxal do $\mathrm{GH}$, conforme ocorreu com a paciente em questão $(12,13)$.

Os valores de $\mathrm{GH}$ ao longo das 24 horas variam entre altos picos e valores muito baixos em indivíduos normais, sendo frequente a presença dos picos noturnos. Nos pacientes acromegálicos as dosagens do GH permanecem relativamente inalteradas, com valores geralmente acima dos normais e sem ritmo circadiano (14). Além disso, são também observados picos esporádicos deste hormônio, sobretudo imediatamente após as refeições $(7,11)$. Observações clínicas mostram que a média dos valores de GH nas 24 horas em indivíduos normais encontra-se abaixo de $2 \mathrm{ng} / \mathrm{ml}$, enquanto valores acima de $10 \mathrm{ng} / \mathrm{ml}$ constituem critério diagnóstico para acromegalia. Valores intermediários podem ser encontrados em outras patologias além da hipersecreção do $\mathrm{GH}$, como tumores carcinóides e diabetes mellitus, entre outras (15).

O teste de estímulo do GH com TRH e com $\mathrm{GnRH}$ que apresenta elevação nos valores de $\mathrm{GH}$ de, pelo menos, $100 \%$ em relação ao basal é considerado inadequadamente elevado. Elevação dos valores de $\mathrm{GH}$ pós TRH já foi evidenciada em outras situações, como na insuficiência renal, na doença hepática crônica, na anorexia nervosa, no hipotireoidismo, na depressão e no diabetes mellitus não insulino dependente (16). Outros estudos demonstraram resultados variáveis neste teste, sendo, por esse motivo, considerado inconsistente para o diagnóstico da acromegalia $(9,16-18)$.

Nos pacientes acromegálicos com níveis séricos elevados de $\mathrm{GH}$ basal, ocorre uma diminuição paradoxal dos valores de GH após estímulo com L-dopa. Todavia, pacientes acromegálicos com valores de $\mathrm{GH}$ basais normais demonstram um aumento dos valores de GH após o estímulo, semelhante ao encontrado nos pacientes normais (19).

O GH estimula a síntese e a secreção do fator de crescimento semelhante à insulina (IGF-I) em diversos sítios do organismo, principalmente no fígado. A depuração de IGF-I na circulação demora algumas horas e este peptídeo se liga predominantemente a proteínas (3). Assim sendo, níveis séricos de IGF-I variam pouco nas 24 horas, refletindo de modo fiel a magnitude da secreção de GH (12). Parece haver uma diferença significativa entre os seus valores nos pacientes normais e naqueles com acromegalia, e, tam- bém, nos pacientes acromegálicos que apresentam valores basais de $\mathrm{GH}$ dentro dos limites da normalidade. No entanto, os níveis de IGF-I são influenciados por vários fatores tais como o estado nutricional, possibilitando resultados falso positivos e falso negativos (6). Valores elevados de IGF-I são encontrados na puberdade e na gravidez, e nestas situações as dosagens deste peptídeo devem ser interpretadas com cautela (20).

$\mathrm{Na}$ acromegalia, os níveis séricos de IGF-I estão invariavelmente elevados e o grau de elevação se correlaciona com as manifestações de hipersomatotropismo. O IGF-I pode permanecer elevado por muitos meses após o GH ter se normalizado após o tratamento, embora o mais comum seja acompanhar a queda dos níveis de $\mathrm{GH}$ (21).

A dosagem da $\mathrm{IGFBP}_{3}$ sérica parece constituir outro método diagnóstico potencialmente útil para a acromegalia. Esta glicoproteina $\mathrm{GH}$ dependente se liga às IGF-I e IGF-II circulantes sendo um marcador sensivel para a hipersecreção do $\mathrm{GH}$, mesmo em pacientes nos quais o $\mathrm{GH}$ encontra-se suprimido para valores abaixo de $2 \mathrm{ng} / \mathrm{ml}$ após a administração oral de glicose (3).

A razão para a presença de níveis séricos basais de $\mathrm{GH}$ dentro dos limites da normalidade em vigência de acromegalia em atividade ainda é motivo de investigação e dúvidas. Mashiter e cols., após estudarem um paciente com grande tumor hipofisário e severo quadro clínico de acromegalia, mas com valores de GH sérico relativamente baixos, sugeriram algumas possibilidades que justificassem tal baixo nível de secreção: 1) alteração na estrutura molecular do $\mathrm{GH}$, com atividade biológica aumentada ou com baixa atividade na dosagem pelo imunoensaio; 2) presença de proteases lisossomais intrapituitárias capazes de degradar grânulos hormonais (inclusive $\mathrm{GH}$ ), causando baixo conteúdo hormonal, uma vez que a atividade do marcador lisossomal $\mathrm{N}$-acetil-- glucosaminidase estava significativamente aumentada neste paciente $(22,23)$.

Campino e cols. isolaram dois tipos de anticorpos da classe $\operatorname{IgG}$ contra o receptor do $\mathrm{GH}$ em pacientes acromegálicos. Uma destas imunoglobulinas interfere com a dosagem de GH sérico feita por radioimunoensaio (hGH RIE), superestimando os níveis séricos deste hormônio. Tal fato pode explicar a persistência de $\mathrm{GH}$ sérico elevado em pacientes acromegálicos submetidos com sucesso a cirurgia transesfenoidal e/ou radioterapia. A outra imunoglobulina detectada parece não interagir com o hGH RIE e, se biologicamente ativa, pode ter participação na gênese dos raros casos de acromegalia nos quais os valores séricos de $\mathrm{GH}$ imunorreativo encontram-se dentro dos limites da normalidade (24). 
Deve-se também lembrar a existência do acromegaloidismo, que são aqueles pacientes que apresentam manifestações clínicas típicas de acromegalia mas nos quais não se consegue demonstrar a presença de tumor hipofisário ou mesmo extrahipofisário produtor de $\mathrm{GH}$. Nestes pacientes, os níveis de $\mathrm{GH}$ e de IGF-I são normais e a resposta aos testes de estímulo são normais (21).

\section{CONCLUSĀO}

Frente às evidências apresentadas, torna-se fundamental a realização de testes dinâmicos de avaliação da regulação do $\mathrm{GH}$ em todo paciente com forte suspeita clínica de acromegalia, mesmo que os exames iniciais não tenham evidenciado hipersecreção de $\mathrm{GH}$. O teste oral de tolerância à glicose parece ser o mais indicado para o diagnóstico, devido à sua facilidade de realização, baixo custo e ausência de risco para o paciente, ainda que a dosagem do IGF-1 e $\mathrm{IGFBP}_{3}$ apresente algum benefício durante a investigação (15). Os demais testes dinâmicos devem ser realizados sempre que necessário e em ambiente hospitalar. A RNM constitui o método de imagem de escolha nestes pacientes.

\section{REFERÊNCIAS}

1. Wajchenberg BL. Tratado de Endocrinologia Clínica. Roca:São Paulo. 1992.

2. Consensus Statement: Benefits versus risks of medical therapy for acromegaly. Am J Med 1994;97:68-73.

3. Melmed S, Ho K, Klibanski A, Reichlin S, Thorner M. Recent advances in pathogenesis, diagnosis and management of acromegaly. J Clin Endocrinol Metab 1995;80:3395-401

4. Rodrigues FF, Violante AHD, Kallas JL, Vaisman $\mathrm{M}$. Acromegalia; aspectos diagnósticos e terapêuticos análise de 18 casos. Arq Neuropsiquiatr 1997;55:85-90.

5. Bates AS, Hoff WV, Jones JM, Clayton RN. An audit of outcome of treatment in acromegaly, Quarterly J Med 1993:86:293-9.

6. Brockmeier SJ, Buchfelder M. Adams EF, Schot W. Fahlbusch R. Acromegaly with normal serum growth hormone levels - clinical features, diagnosis and results of transesfenoidal microsurgery. Horm Metab Res 1992:24:392-6.

7. Mims RB, Bethune JE. Acromegaly with normal fasting growth hormone concentrations but abnormal growth hormone regulation. Ann Int Med 1974;81:781-4.

8. Feingold KR, Goldfine ID, Weinstein PR. Acromegaly with normal growth hormone levels and pituitary histology. J Neurosurg 1979:50:503-7.
9. Shibasaki T, Hotta M, Masuda A, Imaki T, Obara N, Hisuka $N$, et al. Studies on the response of growth hormone (GH)-releasing hormone, thyrotropin-releasing hormone. gonadotropin-releasing hormone, and somatostatin in acromegaly. J Clin Endocrinol Metab 1986;63:167-74.

10. Hanew K, Kokubun M, Sassaki A, Mouri T, Yoshinaga K. The spectrum of pituitary growth hormone responses to pharmacological stimuli in acromegaly. J Clin Endocrinol Metab 1980;51):292-7.

11. Cryer $P E$, Daughaday WH. Regulation of growth hormone secretion in acromegaly. J Clin Endocrinol Metab 1969:29:386-94.

12. Hattori N, Shimatsu A, Kato Y, Koshiyama H, Ishikawa $Y$, Assadian $\mathrm{H}$, et al. Growth hormone response to oral glucose loading measured by highly sensitive enzyme immunoassay in normal subjects and patients with glucose intolerance and acromegaly, J Clin Endocrinol Metab 1990;70:771-7.

13. Beck P, Parker ML, Daughaday WH. Paradoxical hypersecretion of growth hormone in response to glucose. J Clin Endocrinol Metab 1966:26:463-9.

14. Carlson HE, Gillin C, Gorden P, Snyder F. Absence of sleep-related growth hormone peaks in aged normal subjects and in acromegaly. J Clin Endocrinol Metab 1972;34: $1102-8$.

15. Daughaday WH, Starkey RH, Saltman S, Gavin JR, MillsDuniap B, Heath-Monnig E. Characterization of serum growth hormone $(\mathrm{GH})$ and insulin-like growth factor I in active acromegaly with minimal elevation of serum $\mathrm{GH}$. J Clin Endocrinol Metab 1987:65:617-23.

16. Hulting AL, Theodorsson E, Werner S. Thyrotropin-releas ing hormone increases serum levels of growth hormonereleasing hormone and growth hormone in patients with acromegaly. J Intern Med 1992;232:229-35.

17. Ishibashi M, Yamaji T. Effect of thyrotropin-releasing hormone and bromoergocriptine on growth hormone and prolactin secretion in perfused pituitary adenoma tissues of acromegaly. J Clin Endocrinol Metab 1978;47:1251-6.

18. Gelato MC, Merriam GR, Vance ML, Goldman JA, Webb C. Evans WS et al. Effects of growth hormonereleasing factor on growth hormone secretion in acromegaly, J Clin Endocrinol Metab 1985:60:251-7.

19. Tolis $G$, Kovacs L, Friesen H, Martin JB. Dynamic evaluation of growth hormone $(\mathrm{GH})$ and prolactin (hPRL) secretion in active acromegaly with high and low $\mathrm{GH}$ output. Acta Endocrinol 1974;78:2.

20. Gadelha M. Adenohipófise. In: Vaisman $M$, ed. Endocrinologia Clínica. Editora Cultura Médica:Rio de Janeiro; 1998:7-54.

21. Melmed S. Acromegaly, In: Melmed S, ed. The Pituitary. Blackwell Science:Cambridge; 1995:413-42.

22. Mashinter K, De Marco L, Noorden SV, Adams E, Loizou $M$. Joplin GF, et al. Inappropriately low serum GH in acromegalic: lysosomal involvement in intracellular hormone degradation. Metabolism 1982;31:931-6. 
23. Barkan AL, Beltins IZ, Kelch RP. Plasma insulin-like growth factor-1/somatomedin-C in acromegaly: correlation with the degree of growth hormone hypersecretion. J Clin Endocrinol Metab 1988;67:69-73.

24. Campino C, Szecowka J, Lopez JM, Mulchahey J, SerónFerré. Growth hormone $(\mathrm{GH})$ receptor antibodies with GH-like activity occur spontaneously in acromegaly. J Clin Endocrinol Metab 1992;74;751-6.

\section{Endereço para correspondência:}

Mônica Peres da Costa

Praia de icaraí $n^{\circ} 45 / 501$

24.230-000 Niterói, RJ

Fax: $(0 \times \times 21) 719-7897$

e.mail: monicapcosta@hotmail.com 